\title{
Propuesta de modelo de optimización de tiempo de vida útil de depósito de relaves espesados
}

\author{
Proposal model for optimizing the life cycle \\ of thickened tailings deposits
}

\author{
Joaquín Martínez ${ }^{1 *} \quad$ Edwin Franco ${ }^{1}$
}

Recibido 6 de diciembre de 2016, aceptado 26 de marzo de 2018

Received: December 6, 2016 Accepted: March 26, 2018

\begin{abstract}
RESUMEN
Este trabajo propone una metodología de planificación para optimizar el tiempo de vida útil de los depósitos de relaves espesados, específicamente aplicado al depósito de Minera Centinela, perteneciente a Antofagasta Minerals. En él, se utilizaron conceptos metalúrgicos, métodos heurísticos, programación entera y herramientas CAD para la modelación de los relaves en 3D. Para comenzar, se caracterizó geométricamente el depósito, de donde se estimaron principalmente las pendientes de la superficie y las distintas capacidades volumétricas remanentes. Luego, se caracterizó la pulpa de relaves, conociendo sus características reológicas para relacionarlas a una pendiente de disposición. Se trabajó con datos de flujo-metros y análisis de resultados de pruebas de laboratorio. De esta manera, se logró crear una herramienta que calcula el tiempo de vida útil del depósito, ingresando datos de entradas nominales, pesimistas u optimistas. Además, permite conocer el historial de los relaves descargados, facilitando el seguimiento operacional. Los resultados obtenidos determinan la capacidad máxima posible a la que buscaría llegar la planificación al mejorar la geometría de la superficie. Finalmente, se tiene una metodología de planificación, adaptable a condiciones cambiantes, con el objetivo principal de definir volúmenes de relaves espesados a depositar, a partir de las pendientes, capacidades disponibles del depósito y de las características reológicas de los relaves producidos, maximizando la utilización de las distintas áreas del depósito.
\end{abstract}

Palabras clave: Depósitos de relaves espesados, método heurístico, programación entera, relaves, reología.

\begin{abstract}
This work proposes a planning methodology that optimizes the life cycle of thickened tailings deposits, specifically the Centinela Mine thickened tailings deposit owned by Antofagasta Minerals. The methodology included metallurgical concepts, heuristics methods, integer programming and CAD tools for tailings $3 D$ modeling. The thickened tailing deposit was first geometrically characterized, considering its slopes and volumetric capacities in its different sectors. Then, a relationship between the rheological characteristics of the tailings slurry and the beach slope in the deposit was proposed. For this, online data and laboratory tests results analysis were carried out. As a consequence, it was possible to create a tool that calculates the life cycle of the deposit by entering data of nominal, pessimistic or optimistic entries. In addition to that, it allows obtaining the history of the already deposited tailings to facilitate operational follow-up. The results show the maximum possible capacity that could be attained by improving surface shape optimization. Finally, a planning methodology, adaptable to changing conditions is generated, with the
\end{abstract}

1 Escuela de Ingeniería. Universidad Católica del Norte. Larrondo \#281, Guayacán. Coquimbo, Chile.

E-mail: joaquin.me500@gmail.com; edwinfranco@ucn.cl

* Autor de correspondencia 
main objective of defining where to discharge tailings, based on the slopes and available capacities of the deposit and the rheological characteristics of the tailings slurry, thus maximizing the use of the different areas of the deposit.

Keywords: Heuristics methods, integer programming, rheological, tailings, thickened tailings deposits.

\section{INTRODUCCIÓN}

Un desafío muy importante para las faenas mineras, es toda obra constructiva en relación a la disposición de los desechos generados en la producción de minerales [1]. En la industria minera chilena, este tema ha adquirido gran relevancia, ante la disminución de las leyes de los minerales en los yacimientos mineros en explotación [2], obligando a las empresas mineras a extraer grandes toneladas de mineral para poder mantener los niveles óptimos de producción de concentrados. En consecuencia, se ha incrementado la cantidad de desechos que deben ser dispuestos, ya sea como material estéril o en forma de pulpa de relaves [1]. Ante este escenario, se debe tener muy presente los ámbitos técnicos constructivos como los ambientales de la regulación chilena [3].

Además, según el Atlas del agua 2016, elaborado por la Dirección General de Aguas del Ministerio de Obras Públicas [4], se ha presentado un agotamiento en las aguas superficiales y una disminución de los acuíferos, principalmente en zonas del país donde se sitúa la industria minera. Ante la escasez del agua, los permisos para su utilización, son cada vez más estrictos, generando que las mineras innoven en nuevos métodos operacionales, como la utilización de agua de mar, nuevas tecnologías de recuperación de agua y la disposición de los relaves de forma espesada.

A partir de esta situación, se ha generado una gran problemática, dado que se deben crear distintas estrategias, planificaciones a corto, mediano y largo plazo, e implementar tecnologías innovadoras, para una adecuada gestión de los recursos disponibles y para la disposición de los relaves, en forma espesada, sobre la superficie de la tierra.

Este trabajo, va orientado a asistir al personal de planta en determinar secuencias de disposición de los relaves espesados, de manera que se gestionen de manera correcta, en búsqueda de mejorar la superficie y de la maximización del uso de las áreas disponibles para su disposición.

La metodología que se presenta en este trabajo, se desarrolló para ser aplicada al depósito de relaves espesados de Minera Centinela, cuya puesta en marcha fue en el año 2010. Esta minera, es un yacimiento de cobre y oro, ubicado a $30 \mathrm{~km}$ de la localidad de Sierra Gorda, II Región de Antofagasta, en pleno desierto de Atacama, a 2300 metros sobre el nivel de mar (ver Figura 1). Es una de las pioneras en el uso de agua de mar no tratada, para utilizar en sus procesos y fue formada luego de la fusión entre las mineras Esperanza y el Tesoro, siendo respectivamente sus divisiones de sulfuros y óxidos, comenzando a operar como Centinela en noviembre, 2014 [5].

Dentro de las instalaciones consideradas para el objetivo de producción de concentrado de cobre en su división de sulfuros, se encuentra el depósito de relaves espesados (DRE) [6], el cual tiene una capacidad, de diseño inicial, de 750 millones de toneladas de relave. El área máxima del depósito es de aproximadamente 1400 hectáreas. Este depósito se encuentra a $1 \mathrm{~km}$ al noreste de la planta concentradora (ver Figura 2).

El objetivo de este trabajo es aplicar un modelo de optimización de tiempo de vida útil de depósitos de relaves espesados para esto se deben obtener las características geométricas actuales del DRE, modelar el comportamiento de los relaves dispuestos, principalmente de su pendiente de disposición, simulando las distintas formas de descargas de relaves (Canal, sistemas de distribución de relaves, etc.). $Y$ generar alertas ante condiciones operacionales que disminuyan el tiempo de vida útil proyectado según los plazos de operación comprometidos.

Lo que se busca, es generar una herramienta que permita estar preparado ante contingencias ocasionadas por las características de los relaves 
Fuente: Annual Report 2015[5].

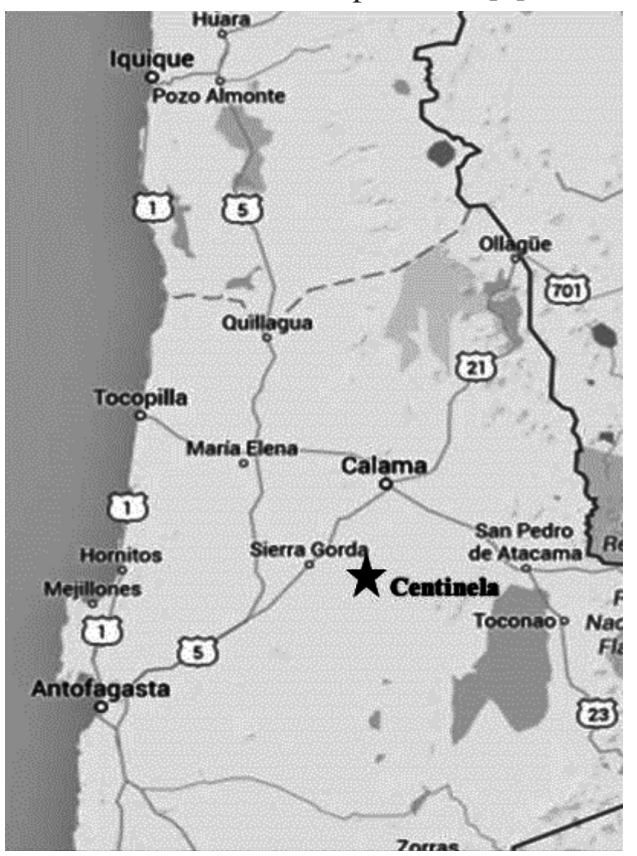

Figura 1. Ubicación geográfica de Minera Centinela, en la II Región de Antofagasta, Chile.

Fuente: Minera Centinela.

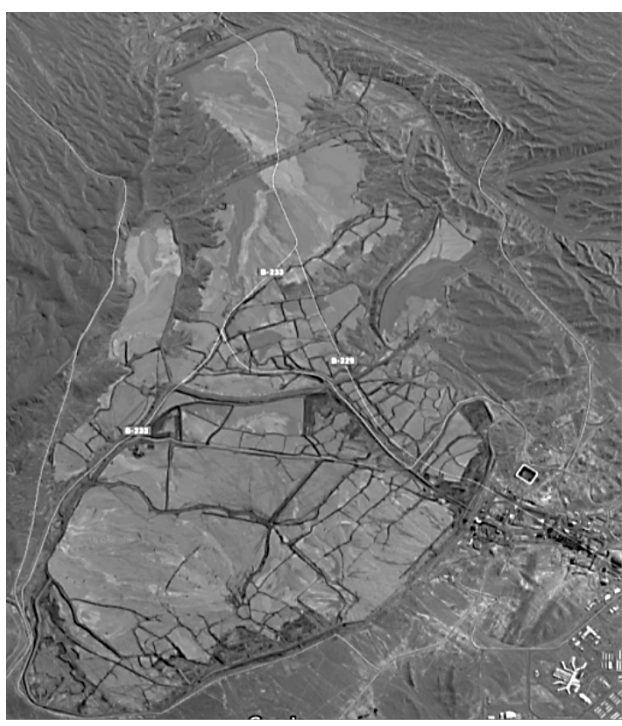

Figura 2. Fotografía satelital del DRE.

[7] o por el estado de las instalaciones y que sea posible conocer cuáles son los sectores apropiados para disponer los volúmenes de relaves enviados desde la planta concentradora [8].

\section{METODOLOGÍA}

Esta metodología, permitirá generar planes de disposición a corto plazo, mediante la simulación de escenarios futuros, previamente a su ejecución.

En primer lugar, se caracteriza geométricamente el depósito. Para realizar esta caracterización, se necesitan conocer sus distintas pendientes y capacidades volumétricas remanentes de los distintos sectores.

Fuente: Elaboración propia.

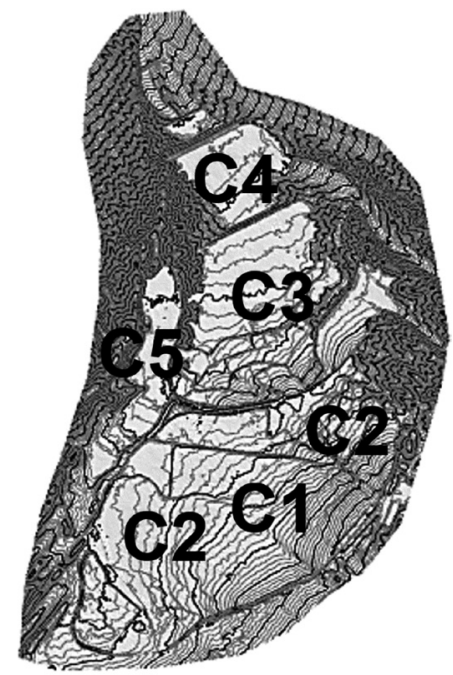

Figura 3. Distribución de sectores del depósito de relaves espesados mediante canchas.

La Figura 3 muestra la distribución de sectores del DRE mediante canchas. Cabe destacar que las canchas 2 son extensiones de la cancha 1 y al observar sus líneas de contornos, da cuenta de las diferencias significativas de pendientes de una extensión a la otra. Todas cuentan con sus correspondientes sistemas de descarga de relaves denominados "peinetas" [1], salvo cancha 4 y cancha 5, cuyos sistemas de descarga funcionan mediante canales para flujos con bajo porcentaje de sólidos. Las "peinetas", tienen el objetivo de distribuir el relave espesado de manera homogénea por toda el área de la cancha respectiva, gracias a los distintos puntos de descargas denominados como "spigots". Estos se encuentran equidistantes a lo largo de todo el sistema para contribuir con la disipación de energía y generación de playas con mayor pendiente, que mejoran la capacidad volumétrica del depósito. 
A continuación, se caracterizan los relaves producidos con la obtención de datos de monitoreo mediante flujo-metros, principalmente el volumen de relaves dispuesto en las distintas canchas y su concentración de sólidos en peso $(\mathrm{Cp})$ respectivo [9]. Para entender la relación entre el contenido de sólidos y la reología del material, se deben enviar diariamente muestras de relaves, obtenidas desde las descargas de los "spigots", a un laboratorio metalúrgico [10]. Los resultados semanales de estas muestras, se analizan y se determina una $\mathrm{Cp}$ y resistencia a la fluencia $(\tau)$ promedio [9].

El objetivo de obtener estos resultados, es relacionar estas variables a una pendiente de disposición de los relaves [1]. El método adecuado para obtener esta relación y modelar los relaves dispuestos, es a partir de $\mathrm{Cp}$ [1], dado que esta variable es posible obtenerla en línea mediante los flujo-metros [9] instalados en las descargas de los espesadores de relaves [11]. Según Sofra [10] "Cambios en la mineralogía y distribución granulométrica en un depósito mineral pueden afectar significativamente la resistencia a la fluencia de los relaves a una concentración de sólidos fija", es por esto que, para una planificación de disposición, se relacionara la pendiente a partir de su resistencia a la fluencia, en Pa.

Tabla 1. Relación estimada de las pendientes, según $\mathrm{Cp}$ y resistencia a la fluencia.

\begin{tabular}{|c|c|c|}
\hline Rangos Cp (\%) & Rangos $\tau$ (Pa) & Pendiente \\
\hline $\mathrm{Cp}<=61$ & $\tau<=8$ & $0,5 \%$ \\
\hline $61<\mathrm{Cp}<=62$ & $8<\tau<=10$ & $1,0 \%$ \\
\hline $62<\mathrm{Cp}<=63$ & $10<\tau<=11$ & $1,5 \%$ \\
\hline $63<\mathrm{Cp}<=64$ & $11<\tau<=15$ & $2,0 \%$ \\
\hline $64<\mathrm{Cp}<=65$ & $15<\tau<=19$ & $2,5 \%$ \\
\hline $65<\mathrm{Cp}<=66$ & $19<\tau<=24$ & $3,0 \%$ \\
\hline $66<\mathrm{Cp}<=67$ & $24<\tau<=31$ & $3,5 \%$ \\
\hline $67<\mathrm{Cp}$ & $31<\tau$ & $4,0 \%$ \\
\hline
\end{tabular}

Fuente: Elaboración propia.

Para calcular el tiempo de vida útil del DRE, se deben conocer las distintas capacidades volumétricas de sus sectores. Luego, se deben proyectar los volúmenes de relaves que se dispondrán, a partir de los siguientes datos de entrada [9]:

- Tonelaje de sólidos, en t/d.

- Densidad del mineral $(\rho m)$, en $\mathrm{t} / \mathrm{m}^{3}$.
- Densidad seca $(\rho s)$, en $\mathrm{t} / \mathrm{m}^{3}$.

- $\quad$ pp promedio, en $\%$.

Según estos datos de entrada, se debe calcular el volumen de relave y el volumen de relave seco [9]. Es este último dato calculado, el que se proyectará en el tiempo, para la estimación de tiempo de vida útil. $\mathrm{El} \mathrm{Cp}$ promedio, corresponde a los $\mathrm{Cp}$ históricamente producidos.

Esta planificación de disposición de relaves espesados, consiste en depositar un volumen de relaves diariamente en los distintos sectores del DRE. Este volumen de relaves, debe ser dispuesto en los sectores adecuados para su contención; por ejemplo, si un relave con una reología que produzca una pendiente del orden de $0.5 \%$ es dispuesto en un sector de pendientes del orden de $2.0 \%$ causará daños en su geometría canalizando los flujos de relaves espesados hacia zonas inadecuadas, agotando la capacidad del sector anticipadamente. Una buena asignación de los sectores espera mejorar la geometría mediante la generación de playas y principalmente la maximización de la utilización de las áreas de depositación del DRE.

Para realizar este plan, se deben considerar los siguientes requerimientos y condiciones operativas:

- El volumen de relaves espesados a disponer debe ser asignado a una determinada cancha con la pendiente adecuada para su contención.

- El volumen de relaves espesados a disponer debe ser asignado a una determinada cancha con capacidad volumétrica disponible suficiente para su contención.

- Las capacidades de los sectores varían dependiendo de los desplazamientos futuros de los sistemas de descarga de relaves espesados.

- Sólo se puede asignar una cancha a la vez, es decir no pueden ser programadas dos o más canchas simultáneamente.

- Una cancha no puede volver a ser asignada sin que antes se hayan cumplido sus horas de secado correspondientes.

- Los sistemas de descarga de relaves por "peinetas" están diseñados para descargar flujos del orden de Cp 62-67\%.

- Para los flujos de bajos sólidos, es decir, con un Cp menor a 62\%, se deben asignar las Canchas 4 y 5 , utilizando los canales de descarga. 
- Los canales de descarga no deben ser utilizados para flujos superiores a una Cp 62\%.

- Sólo se puede asignar una determinada cancha, si sus sistemas o canales de descarga se encuentran operativos.

Para dar solución al problema presentado, se analiza si los requerimientos pueden ser satisfechos o no; y dada las distintas contingencias que se pueden producir en una planta concentradora, resulta poco posible encontrar una solución óptima para la planificación de disposición de relaves espesados mediante un método científico que satisfaga todos los requerimientos. Además, dada las contingencias que se pueden presentar, se decidió realizar la planificación de manera semanal, la cual se puede replicar para todo el mes si no se presentan contingencias importantes. Por esto se decidió utilizar el método heurístico de la IO, que describe Hillier [14] para determinar las horas óptimas de disposición y asignación de canchas, según sus capacidades volumétricas disponibles para la contención de los relaves espesados producidos. Para encontrar la mejor solución, se determina previamente que sistemas de descargas se utilizarán, además de la cantidad de "spigots" operativos. También se determina que las horas de secado del relave corresponderán como mínimo las horas de disposición que tuvo cada cancha $(1: 1)$. De esta manera, se obtendrá la mejor utilización a los sectores del DRE.

Una vez conocidas las horas y volúmenes óptimos a descargar en las distintas canchas, se deben definir intervalos de descargas. La necesidad de disponer los volúmenes totales definidos por canchas mediante intervalos, genera una alteración en los volúmenes finales que se dispondrán por canchas. Para cumplir el objetivo de disponer a partir de las capacidades disponibles, surge la problemática de reducir esta diferencia de volúmenes que alteran los resultados esperados. Para esto, se ha desarrollado un modelo de programación entera [14], el que busca definir las horas óptimas de los intervalos de disposición que minimicen las diferencias de volúmenes finales con respecto al inicial calculado. Antes de definir el modelo, primero se debe definir la notación utilizada.

$\boldsymbol{x i}$ : Horas de los intervalos de disposición en la cancha $\mathrm{i}$, para $\mathrm{i}=1,2,3,4,5$.

$\boldsymbol{h s}$ : Horas semanales. $\boldsymbol{h} \boldsymbol{A}$ : Horas mínimas de intervalo de disposición por canchas.

$\boldsymbol{h} \boldsymbol{B}:$ Horas máximas de intervalo de disposición por canchas.

$\boldsymbol{v} \boldsymbol{h}$ : Volumen por hora.

$\boldsymbol{k} \boldsymbol{i}$ : Porcentaje de las horas a disponer inicial en la cancha i.

$\boldsymbol{v i} \mathbf{j}$ : Volumen a disponer $\mathrm{j}$ en la cancha $\mathrm{i}$, para $\mathrm{j}=$ 1, 2 (inicial y final, respectivamente).

$$
v_{i j}=h_{i j} * v_{h}
$$

$\boldsymbol{h i j}$ :Horas de utilización i de la cancha j.

$$
h_{i j}=x_{i} * c_{i}
$$

ci: Cantidad de ciclos de disposición en la cancha i.

$$
c_{i}=\frac{h_{s}}{\left(x_{i}+y_{i}\right)}
$$

$\boldsymbol{y} \boldsymbol{i}$ : Horas de secado de la cancha i hasta que vuelva a utilizarse.

$$
y_{i}=\left(\sum_{i=1}^{5} x_{i}\right)=x_{i}
$$

$\boldsymbol{\varphi}:\left\{\begin{array}{c}1 \text { si } v_{i 1}>0, \\ 0 \text { de otra manera; }\end{array}\right.$

Con la notación definida, el modelo de programación entera es el siguiente:

$$
\text { Minimizar } Z=\sum_{i=1}^{n}\left|v_{i 2}-v_{i 1}\right|
$$

Sujeto a

$$
\begin{gathered}
\left(\sum_{i=1}^{s} x_{i}\right)-x_{i} \geq x_{i} ; \forall i \\
x_{i} \geq h_{A} * \varphi ; \forall i \\
x_{i} \leq h_{B} * \varphi ; \forall i \\
x_{i} \leq h_{i 1} * k_{i} ; \forall i \\
\varphi \in\{0,1\}
\end{gathered}
$$

$x_{i}$ son enteros, para $\mathrm{i}=1,2,3,4,5$.

La función objetivo representa la minimización de las diferencias absolutas entre el volumen final 
dispuesto mediante intervalos y el volumen óptimo inicial calculado. La restricción (6) garantiza que se cumplan las horas de secado mínimos de cada cancha, de esta manera los cruces de las horas de los intervalos de disposición y sus horas de secado serán factibles, es decir, en ningún momento el modelo estará incapacitado de asignar alguna cancha por el incumplimiento de las horas de secado correspondientes. La restricción (7) y (8) evitan que se determinen horas de intervalos menores y mayores a las designadas respectivamente (si el volumen inicial calculado a depositar por cancha es superior a 0). La restricción (9) acota la solución de intervalos de horas, que deben ser menores o iguales al porcentaje $\mathrm{k}$ de las horas asignadas a cada cancha. Este modelo se modelará en el solucionador básico de Excel, Solver.

Una vez conocidos los volúmenes finales a depositar por cada cancha y la cantidad de intervalos semanales, el modelo entregará un ciclo de disposición, el cual se replicará a continuación de la finalización de este, hasta el término de la semana. Se designó una preferencia por canchas con mayores horas de intervalos de disposición y luego, si tienen igual cantidad de horas, basarse en su orden numérico. Para resolver este problema, se ha programado una aplicación para priorizar de manera automática los ciclos de depositación, considerando el requerimiento de programar solo una cancha a la vez y con la posibilidad de reemplazar una cancha asignada por otra, si se requiere, para que el plan sea flexible y adaptable a condiciones cambiantes.

A continuación, se modelaron los resultados con el software Muk3D, para conocer qué áreas del depósito ocuparán finalmente estos volúmenes planificados y el espesor de capa aproximado que tendrán al ser depositados sobre la superficie. Para esto resulta necesario conocer las distintas canalizaciones presentes en el depósito de relaves, para que la modelación de los volúmenes siga sus trayectorias como líneas de descargas. Como producto final, se obtiene un horario de disposición, además de la representación gráfica de los resultados esperados en 3D.

Los puntos anteriores describen cómo se llevará a cabo la planificación de disposición de relaves espesados. Para darle continuidad a este trabajo, se debe realizar un seguimiento operacional de los resultados esperados. Para esto se deben comparar los resultados planificados versus los resultados reales, de esta manera se podrá validar esta metodología a partir de calibraciones continuas.

\section{DESARROLLO}

La Figura 4, grafica un ejemplo de las pendientes obtenidas, específicamente, de una sección tomada en el sector de cancha 3. Dada las diferencias significativas de las pendientes en algunas canchas, como por ejemplo las canchas 2, y la manera en que llega el relave hacia ellas, se necesita seccionar el DRE mediante zonas de disposición.

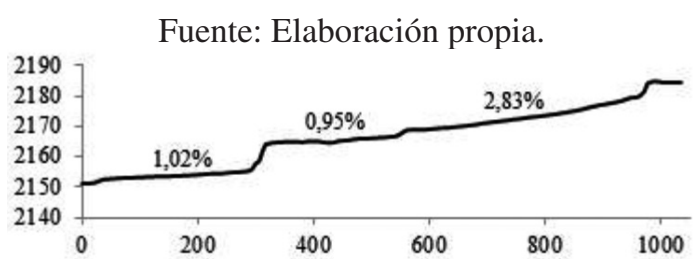

Figura 4. Sección de pendientes Cancha 3, distancia (m) versus elevación $(\mathrm{m})$.

Fuente: Elaboración propia.

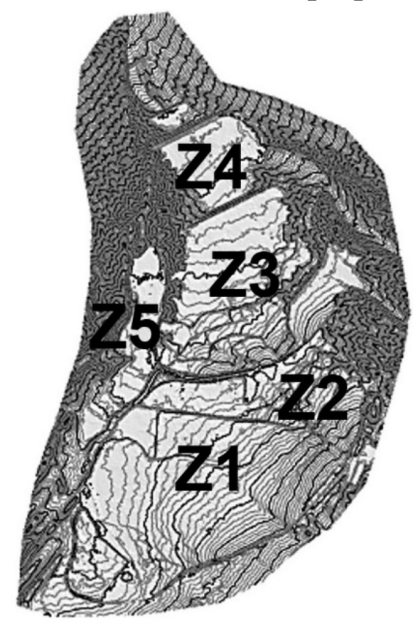

Figura 5. Zonas de depositación en el DRE.

La Figura 5, presenta una nueva distribución de sectores mediante cinco zonas. La cancha 1 se unirá a su extensión izquierda, denominándose zona 1 y su extensión derecha zona 2. Las zonas 3, 4 y 5, seguirían siendo similares a su anterior distribución por canchas. De esta manera se facilitará el posterior cálculo de capacidades volumétricas. 
A continuación, se crea una metodología de seis etapas con diferentes pasos que se describen a continuación:

Etapa 1: Obtener un levantamiento topográfico periódico, el cual permita trabajar en las siguientes etapas.

Etapa 2: Comparar la nueva topografía con la topografía anterior, como primera visualización de los resultados de la planificación. Caracterizar los relaves a partir de los resultados obtenidos de las muestras enviadas al laboratorio metalúrgico correspondiente. Luego, se calcula el volumen de relaves a disponer y se relaciona a una pendiente de disposición, según Tabla 1. Finalmente se modelan los relaves ya dispuestos, de manera de actualizar la superficie topográfica y utilizarla como línea base para calcular las capacidades volumétricas del DRE.

Etapa 3: Calcular las distintas capacidades de las zonas de disposición y posteriormente se calcula el tiempo de vida útil.

Etapa 4: Generar plan de disposición semanal mediante los siguientes siete pasos:

1. Determinar el volumen semanal de relave a descargar y su pendiente de disposición. La pendiente de disposición, se utiliza de manera fija para toda la planificación semanal.

2. Seleccionar los sistemas de descarga que se utilizarán y la cantidad de "spigots" operativos en cada uno de ellos, para así tener en cuenta las condiciones operativas anteriormente expuestas.

3. Indicar los resultados de capacidades volumétricas de las zonas de disposición para el relave planificado y las horas de disposiciones semanales por cada una de ellas.

4. Indicar los volúmenes y horas a disponer, volviendo a la distribución anterior por canchas.

5. Obtener los resultados del modelo de programación entera, el cual determina las horas de intervalos de disposición óptimos por canchas, minimizando la diferencia entre el volumen final y el volumen óptimo inicial indicado en el paso 4, respetando las distintas restricciones.

6. Realizar la programación de los intervalos anteriores, obteniendo como resultado un horario de disposición que cumpla con el requerimiento de descargar en solo una cancha simultáneamente.

7. Modelar los volúmenes planificados.

Etapa 5: Conducir un seguimiento operacional, donde se deben analizar los resultados obtenidos versus los planificados, diarios y semanalmente, mediante reportes operacionales. Además, se deben actualizar las capacidades según los volúmenes descargados.

Etapa 6: Indicar requerimientos adicionales y calibración de metodología.

La Figura 6, muestra la metodología con un enfoque cíclico, favoreciendo el mejoramiento continuo del plan de disposición [12]. El plan debe seguir

Fuente: Elaboración propia.

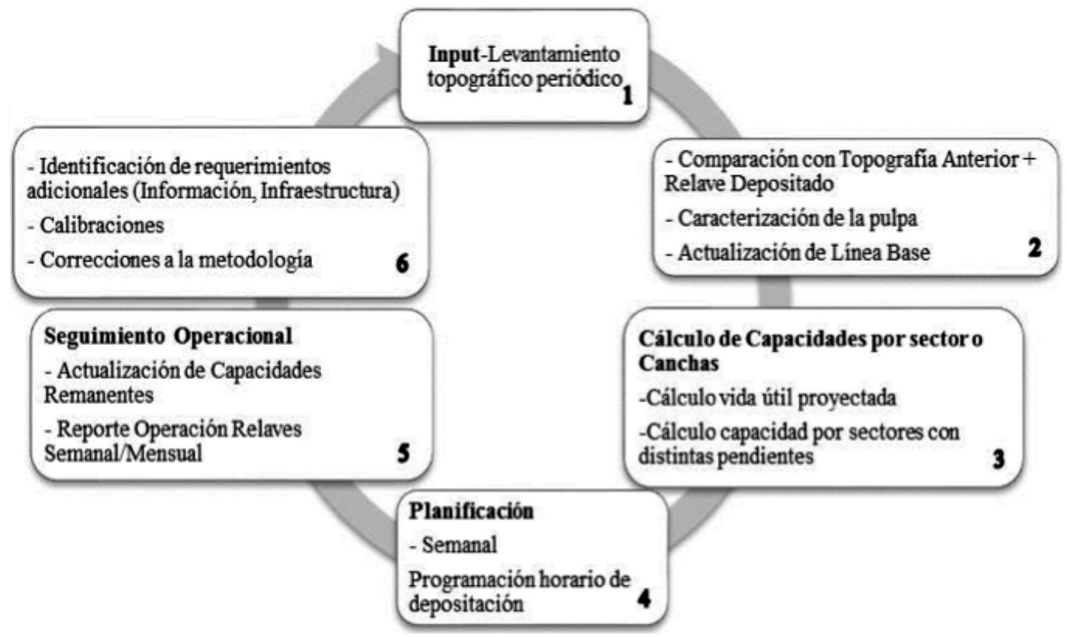

Figura 6. Metodología plan de disposición. 
la estructura de esta metodología para maximizar el tiempo de vida útil del DRE a partir de la mejor utilización de los sectores.

\section{RESULTADOS}

A continuación, se muestran los resultados de las diferentes etapas:

Resultados Etapa 2: Se caracterizó el relave espesado con una $\mathrm{Cp}$ y resistencia a la fluencia promedio de $63.06 \%$ y $10.01 \mathrm{~Pa}$, respectivamente. Según la Tabla 1, la pendiente de disposición será de $1.5 \%$.

Resultados Etapa 3: El tiempo de vida útil del DRE se muestra en la Figura 7. Las diferentes curvas representan los desplazamientos futuros de los sistemas de descarga. Se puede observar, como aumenta la capacidad al incrementar la pendiente de disposición y al desplazar los sistemas de descargar del DRE.

Se deja en evidencia, las acciones que se deberían tomar, si se requiere un aumento de las capacidades volumétricas. La Tabla 2, muestra las capacidades acumuladas para las distintas pendientes y desplazamientos de los sistemas de descarga.

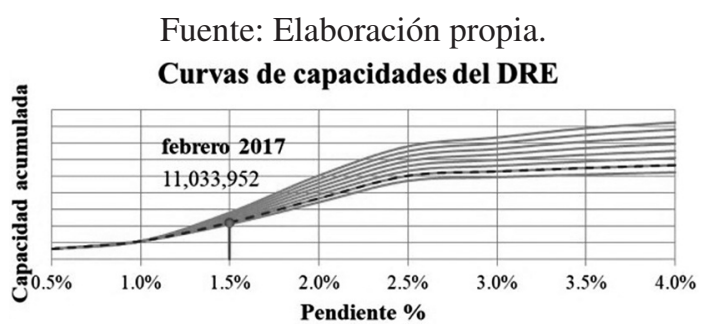

Figura 7. Tiempo de vida útil del DRE para la capacidad actual $\left(\mathrm{m}^{3}\right)$.

- Resultados Etapa 4: Se descargarán $693036 \mathrm{~m}^{3}$ de relave de manera semanal, con una pendiente de $1,5 \%$ y se utilizarán los sistemas de descarga por "peinetas". La Tabla 3, muestra los volúmenes disponibles, se indica que la zona 2 no tiene capacidad disponible para la pendiente a utilizar y no se utilizará la zona 5, dada las condiciones operativas, de utilizar los canales de descargas solo para flujos de bajos sólidos.

La Tabla 4, muestra las horas semanales en que se debe descargar relave por cada cancha
Tabla 2. Capacidades acumuladas del DRE (millones de $\mathrm{m}^{3}$ ).

\begin{tabular}{|c|c|c|c|c|c|c|c|c|}
\hline 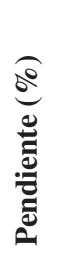 & 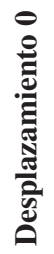 & 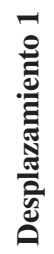 & 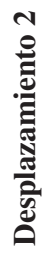 & 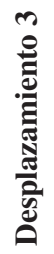 & 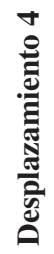 & 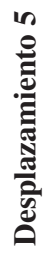 & 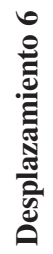 & 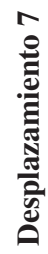 \\
\hline 0,5 & 3 & 3 & 3 & 3 & 3 & 3 & 3 & 3 \\
\hline 1,0 & 5 & 5 & 5 & 5 & 5 & 5 & 5 & 5 \\
\hline 1,5 & 10 & 11 & 11 & 12 & 12 & 13 & 13 & 14 \\
\hline 2,0 & 17 & 18 & 19 & 20 & 21 & 23 & 24 & 25 \\
\hline 2,5 & 23 & 25 & 26 & 28 & 29 & 31 & 32 & 34 \\
\hline 3,0 & 24 & 26 & 28 & 30 & 31 & 33 & 35 & 36 \\
\hline 3,5 & 25 & 27 & 29 & 31 & 33 & 35 & 37 & 39 \\
\hline 4,0 & 26 & 28 & 30 & 32 & 34 & 37 & 39 & 41 \\
\hline
\end{tabular}

Fuente: Elaboración propia.

Tabla 3. Volúmenes disponibles de las zonas, según características del relave.

\begin{tabular}{|c|c|c|c|c|}
\hline 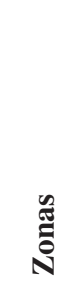 & 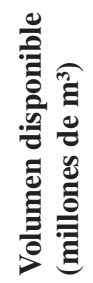 & 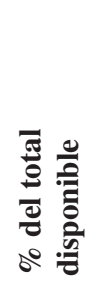 & 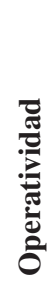 & 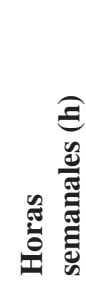 \\
\hline $\mathrm{Z} 1$ & 2,58 & $28 \%$ & 1 & 47 \\
\hline $\mathrm{Z} 2$ & 0 & $0 \%$ & 1 & 0 \\
\hline $\mathrm{Z} 3$ & 5,47 & $58 \%$ & 1 & 97 \\
\hline $\mathrm{Z} 4$ & 1,32 & $14 \%$ & & 24 \\
\hline Z5 & 1,79 & $0 \%$ & & 0 \\
\hline Total & 9,38 & $100 \%$ & & 168 \\
\hline
\end{tabular}

Fuente: Elaboración propia.

Tabla 4. Volúmenes óptimos a depositar por canchas.

\begin{tabular}{|c|c|c|}
\hline Canchas & $\begin{array}{c}\text { Horas semanales } \\
\text { (h) }\end{array}$ & $\begin{array}{c}\text { Volumen } \\
\left({ }^{(} \mathbf{0 0 0} \mathbf{~ m}^{\mathbf{3}}\right)\end{array}$ \\
\hline $\mathrm{C} 1$ & 36 & 148.50 \\
\hline $\mathrm{C} 2$ & 18 & 74.25 \\
\hline $\mathrm{C} 3$ & 114 & 470.27 \\
\hline $\mathrm{C} 4$ & 0 & 0 \\
\hline $\mathrm{C} 5$ & 0 & 0 \\
\hline Total & 168 & 693.03 \\
\hline
\end{tabular}

Fuente: Elaboración propia. 
y el volumen relacionado a esta cantidad de horas. De esta manera, se obtiene un volumen óptimo a disponer por canchas, a partir de las capacidades volumétricas de cada sector, para las características del relave producido.

El resultado del modelo de programación entera se muestra en la Tabla 5.

Tabla 5. Resultados modelo de programación entera.

\begin{tabular}{|c|c|c|c|c|c|c|}
\hline & 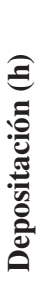 & 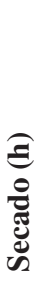 & $\frac{\tilde{0}}{0}$ & 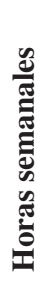 & 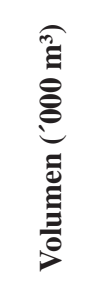 & 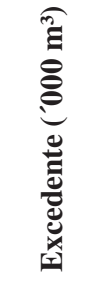 \\
\hline C 1: & 4 & 8 & 14,0 & 56 & 231,01 & 82,50 \\
\hline C 2: & 2 & 10 & 14,0 & 28 & 115,50 & 41,25 \\
\hline C 3: & 6 & 6 & 14,0 & 84 & 346,51 & 123,75 \\
\hline C 4: & 0 & 12 & 14,0 & 0 & 0 & 0 \\
\hline \multirow[t]{2}{*}{ C 5: } & 0 & 12 & 14,0 & 0 & 0 & 0 \\
\hline & & & 14,0 & & F.O. & 247,51 \\
\hline
\end{tabular}

Fuente: Elaboración propia.

La Figura 8, muestra los resultados de la disposición de los volúmenes planificados para una pendiente de $1.5 \%$. De esta manera,

Fuente: Elaboración propia.
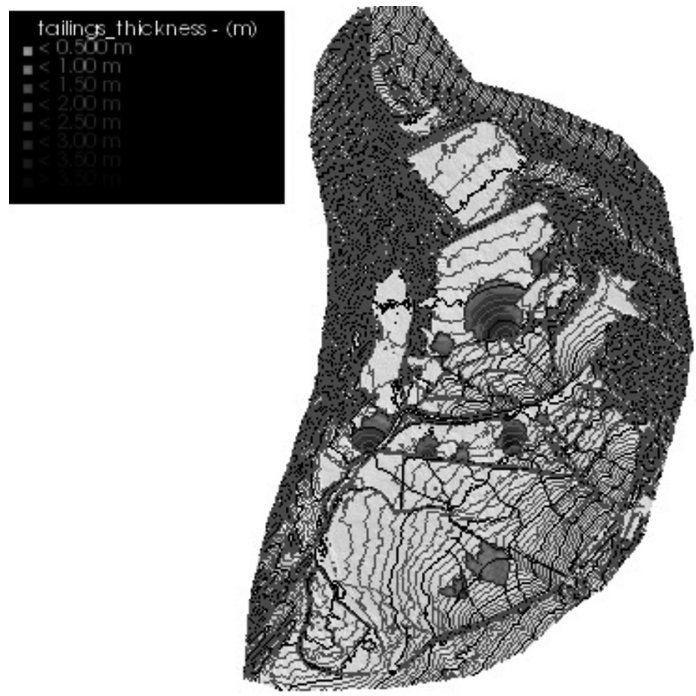

Figura 8. Resultado plan de depositación. se conocen las áreas que ocuparán los relaves y las distintas canalizaciones que se presentarán en el DRE.

\section{CONCLUSIONES}

Este trabajo busca ser un aporte a los nuevos desafíos de la industria minera, como uno de los puntos de partida en el desarrollo de las técnicas de disposición de los relaves espesados. Se logra entender el comportamiento de los relaves sobre la superficie y se utiliza este conocimiento para asignar los sectores adecuados. Es importante destacar los beneficios que se obtienen mediante la implementación de este modelo y el rol fundamental que cumple la utilización del software Muk3D.

I. Minimiza la incertidumbre de la eficiencia de la operación del depósito. Permite hacer rápidos análisis de la evolución de la geometría de la superficie y diferentes simulaciones de escenarios operacionales, como, por ejemplo, ante incrementos o disminución de la pendiente de disposición.

II. Permite anticiparse a distintas contingencias operacionales, al tener la posibilidad de tomar acciones necesarias para mantener el relave en las zonas deseadas, evitando canalizaciones que puedan producir daños en la geometría del depósito.

III. Se mejora la eficiencia de la planificación, al crear una herramienta que permite la programación de los volúmenes planificados en un tiempo reducido y de manera flexible, adaptable a condiciones cambiantes.

IV. Se optimiza el tiempo de vida útil del depósito, mediante una técnica cíclica de mejoramiento continuo, que favorece la generación de playas para mejorar la geometría de la superficie.

Este modelo de planificación, al poseer un enfoque cíclico, necesita ser un proceso permanente y continuo y así su ejecución pueda ser evaluable y medible. Se recomienda la creación de distintos indicadores que evalúen el desempeño mediante esta metodología propuesta y la manera actual de planificación de las deposiciones de relaves. Uno de los principales indicadores son los siguientes: 
- Desviaciones diarias, semanales y mensuales del plan de depositación.

- Pendiente promedio de crecimiento de las cubetas del depósito.

- Distribución de pendientes en el depósito de relaves.

Con el objetivo de generar una discusión sobre la eficiencia de la utilización de los distintos sectores y dar consistencia al desempeño de los trabajos relacionados con el DRE, se sugiere presentar de manera periódica los siguientes puntos a toda la organización involucrada.

- Capacidades actuales del depósito de relaves espesados.

- Resultados obtenidos de la depositación de la semana anterior.

- Actualización de las características del depósito.

- Plan de depositación (programación horaria).

- Modelación de resultados esperados.

Este trabajo deja espacios para seguir investigando, por ejemplo, la calibración de las pendientes de disposición con respecto a la reología y los supuestos que ello implica en la planificación semanal.

\section{AGRADECIMIENTOS}

A los ingenieros de Minera Centinela, Carlos Calderón y Erwin Cortés, por su disposición y por compartir los conocimientos necesarios para realizar este trabajo. A MineBridge Software, por todo el apoyo en el desarrollo de las modelaciones obtenidas.

\section{REFERENCIAS}

[1] N. Ramírez. "Guía técnica de operación y control de depósitos de relaves". pp. 3-20. 2007. Fecha de consulta: 10 de enero de 2016. URL: http://www. sernageomin.cl/pdf/mineria/seguridad/ GuiaTecOperacionDepRelaves.pdf

[2] Consejo minero. "Chile y la minería: Éxitos y desafíos compartidos". 2013. Fecha de consulta: 23 de febrero de 2016. URL: http://www.consejominero.cl/wp-content/ uploads/2013/04/Chile-y-la-mineria_Exitosy-desafios-compartidos2_dic2012.pdf

[3] D. Vio. Reglamento para la aprobación de proyectos de diseño, construcción, operación y cierre de los depósitos de relaves. pp. 1-18.
2007. Fecha de consulta: 24 de febrero de 2016. URL: http://www.sonami.cl/site/wp-content/ uploads/2015/10/Decreto-N\%C2\%B0-248Reglamento-para-la-aprobaci\%C3\%B3nde-proyectos-de-dise \% C 3\%B 1 oconstrucci\%C3\%B3n-operaci\%C3\%B3n-ycierre-de-tranques-de-relaves-1.pdf

[4] C. Estévez. "Atlas del agua, Chile 2016", pp. 90-103. 2016. Fecha de consulta: 27 de noviembre de 2016. URL: http://www. dga.cl/DGADocumentos/Atlas2016parte317marzo2016b.pdf

[5] Antofagasta PLC. Annual Report 2015, pp. 43-44. Fecha de consulta: 25 de mayo de 2016. URL:http://www.antofagasta.co.uk/

[6] E. Robinsky. "Tailings dam failure need not be disasters - the thickened tailings disposal (TTD) system". CIM bulletin. Montreal, Canada. 1999.

[7] D. Boger, P. Scales y F. Sofra. "Rheological concepts". Paste and thickened tailings. Perth, Australia. 2002.

[8] Sonami. Etapas del proceso productivo de una mina. pp. 5-30. 2012. Fecha de consulta: 15 de diciembre de 2016. URL: http://www.sonami.cl/site/wp-content/ uploads/2016/04/01.-Etapas-del-ProcesoProductivo-de-una-Mina.pdf

[9] R. Giles. "Mecánica de los fluidos e hidráulica". McGraw-Hill. Tercera edición. Madrid, España. pp. 1-167. 1994.

[10] F. Sofra. "Rheological assessment - A road map for plant designers and operator". Ninth international seminar on paste and thickened tailings. Limerick, Ireland. 2006.

[11] B. Wills. "Mineral processing technology". Butterworth-Heinemann. Séptima edición. Oxford, UK. pp. 378-389. 2006.

[12] M. Bernal. La planificación: Conceptos básicos, principios, componentes, características y desarrollo del proceso. pp. 2-5. 2012. Fecha de consulta: 19 de julio de 2016. URL: https:// nikolayaguirre.files.wordpress.com/2013/04/1introduccic3b3n-a-la-planificacic3b3n1.pdf [13] Itescam. "Introducción a la reología". 2010. 23 de septiembre de 2016. URL: https:// www.itescam.edu.mx/principal/sylabus/ fpdb/recursos/r53208.DOC

[14] F. Hillier y G. Lieberman. "Introducción a la investigación de operaciones". McGrawHill. Novena edición. Ciudad de México, México. pp. 428-600. 2010. 